\title{
On the (non-)Contractibility of the Order Complex of the Coset Poset of an Alternating Group
}

\author{
Massimiliano Patassini
}

ABSTRACT - Let Alt $k$ be the alternating group of degree $k$. In this paper we prove that the order complex of the coset poset of Alt $_{k}$ is non-contractible for a big family of $k \in \mathbb{N}$, including the numbers of the form $k=p+m$ where $m \in\{3, \ldots, 35\}$ and $p>k / 2$. In order to prove this result, we show that $P_{G}(-1)$ does not vanish, where $P_{G}(s)$ is the Dirichlet polynomial associated to the group $G$. Moreover, we extend the result to some monolithic primitive groups whose socle is a direct product of alternating groups.

Mathematics Subject Classification (2010). 20D30, 20P05, $11 \mathrm{M} 41$.

KEYwords. Probabilistic zeta functions; simplicial complexes; order complexes; contractibility; coset posets; alternating groups; Brown conjecture.

\section{Introduction}

For a finite group $G$, let $\mathcal{C}(G)$ be the set of (right) cosets of all proper subgroups of $\mathrm{G}$, partially ordered by inclusion. Let $\Delta=\Delta(C(G))$ be the order complex of $\mathcal{C}(G)$, so the $k$-dimensional faces of $\Delta$ are chains of length $k$ from $\mathcal{C}(G)$. The study of $\Delta$ was initiated in a paper of K. S. Brown (see [2]), who attributed therein to S. Bouc the observation that there exists a connection between the reduced Euler characteristic $\tilde{\chi}(\mathcal{C}(G))$ and the Dirichlet polynomial $P_{G}(s)$ of $G$, defined by

$$
P_{G}(s)=\sum_{n=1}^{\infty} \frac{a_{n}(G)}{n^{s}}, \text { where } a_{n}(G)=\sum_{H \leq G,|G: H|=n} \mu_{G}(H) .
$$

Here $\mu_{G}$ is the Möbius function of the subgroup lattice of $G$, which is

(*) Indirizzo dell'A.: Via Rovede, 13, 31020 Vidor (TV), Italy.

E-mail: frapmass@gmail.com 
defined inductively by $\mu_{G}(G)=1, \mu_{G}(H)=-\sum_{K>H} \mu_{G}(K)$. The counterpart of the Dirichlet polynomial is called the probabilistic zeta function of $G$ (see [1] and [6]).

In particular, Brown ([2], § 3) showed that

$$
P_{G}(-1)=-\tilde{\chi}(\mathcal{C}(G)) \text {. }
$$

It is a well-known fact that if $\Delta(\mathcal{C}(G))$ is contractible, then its reduced Euler characteristic $\tilde{\chi}(\mathcal{C}(G))$ is zero. Hence, if $P_{G}(-1) \neq 0$, then the simplicial complex associated to the group $G$ is non-contractible.

Moreover, in [2], Brown conjectured the following.

Conjecture 1. If $G$ is a finite group, then $P_{G}(-1) \neq 0$. Hence the order complex of the coset poset of $G$ is non-contractible.

The conjecture was proved for some families of groups, as the following theorem shows.

THEOREM 2. Let $G$ be a finite group.

(1) If $G$ is soluble, then $P_{G}(-1) \neq 0$ (see [2]).

(2) If $G$ is a classical group which does not contain non-trivial graph automorphisms, then $P_{G}(-1) \neq 0$ ([9, Theorem 2]).

(3) If $G$ is a Suzuki simple group or a Ree simple group, then $P_{G}(-1) \neq 0([7])$.

Moreover, in our PhD thesis (see [8, Theorem 7.1]), we proved Conjecture 1 for a class of monolithic primitive groups with socle isomorphic to a direct product of copies of a simple classical group with some conditions on the rank and the number of factors of the direct product.

In this paper we show the following.

Theorem 3. Let $G$ be $\mathrm{Sym}_{k}$ or $\mathrm{Alt}_{k}$. Let $p$ be a prime number such that $\frac{k}{2}<p<k-2$.

(1) If $k-p \leq 35$, then $P_{G}(-1) \neq 0$.

(2) If $p>2((k-p) \text { ! })^{3}$, then $P_{G}(-1) \neq 0$.

A proof of this result proceeds as follows (the general strategy is the same which we employed in [9]). Let $r$ be a prime number and let $f(s)=\sum_{n \geq 1} \frac{a_{n}}{n^{s}}$ be a Dirichlet polynomial. We denote by $f^{(r)}(s)$ the Dirichlet 
polynomial

$$
\sum_{(n, r)=1} \frac{a_{n}}{n^{s}}
$$

Let $p$ be a prime number such that $\frac{k}{2}<p<k-2$. We have that

$$
P_{G}(s)=P_{G}^{(p)}(s)+\sum_{p \mid k} \frac{a_{k}(G)}{k^{s}} .
$$

The first summand $P_{G}^{(p)}(s)$ collects the contribution given by the subgroups $H$ of $X$ such that $H$ contains a Sylow $p$-subgroup, which are intransitive subgroups of $G$ (see [10, Lemma 9]). In [10, Proposition 12] an explicit formula for the Dirichlet polynomial $P_{G}^{(p)}(s)$ is given. So, a careful analysis of the value $P_{G}^{(p)}(-1)$, shows that $\left|P_{G}^{(p)}(-1)\right|_{p}=p$ in many cases (see Section 3 ), where $|a|_{p}$ is the greatest power of $p$ that divides the integer number $a$ (see Section 2 for a more precise definition).

Since $n$ divides $a_{n}(G)$ for $n \in \mathbb{N}-\{0\}$ (see Lemma 5), we have that

$$
\left|\sum_{p \mid n} a_{n}(G) n\right|_{p} \geq p^{2}
$$

hence

$$
\left|P_{G}(-1)\right|_{p}=\left|P_{G}^{(p)}(-1)+\sum_{p \mid n} a_{n}(G) n\right|_{p}=p
$$

whenever $\left|P_{G}^{(p)}(-1)\right|_{p}=p$. So we conclude $P_{G}(-1) \neq 0$.

With a little more work we can obtain a more general result. Let us note that if $N$ is a normal subgroup of a finite group $G$, then $P_{G}(s)=P_{G / N}(s) P_{G, N}(s)$ (see [2]), where

$$
P_{G, N}(s)=\sum_{n \geq 1} \frac{a_{n}(G, N)}{n^{s}}, \text { with } a_{n}(G, N)=\sum_{\substack{H \leq G, G \in: H \mid=n, N H=G,}} \mu_{G}(H) .
$$

Thus, if $1=N_{0}<N_{1}<\ldots<N_{l}=G$ is a chief series of $G$, applying the above formula repeatedly, we obtain

$$
P_{G}(s)=\prod_{i=0}^{l-1} P_{G / N_{i}, N_{i+1} / N_{i}}(s) .
$$

If the chief factor $N_{i+1} / N_{i}$ is abelian, by a result of [4], we have that

$$
P_{G / N_{i}, N_{i+1} / N_{i}}(s)=1-\frac{c_{i}}{\left|N_{i}\right|^{s}}
$$


where $c_{i}$ is the number of complements of $N_{i+1} / N_{i}$ in $G / N_{i}$. Hence $P_{G / N_{i}, N_{i+1} / N_{i}}(-1) \neq 0$.

If the chief factor $N_{i+1} / N_{i}$ is non-abelian, then there exists a monolithic primitive group $L_{i}$ such that

$$
P_{L_{i}, \mathrm{soc}\left(L_{i}\right)}^{(r)}(s)=P_{G / N_{i}, N_{i+1} / N_{i}}^{(r)}(s)
$$

for each prime divisor $r$ of the order of the socle $\operatorname{soc}\left(L_{i}\right)$ of $L_{i}$ (see, for example, [3, Proposition 16]).

The above discussion suggests that in order to prove Conjecture 1, we can focus our attention on the monolithic primitive groups. In particular, here we prove the following.

THeOREm 4. Let $G$ be a primitive monolithic group with socle $N$ isomorphic to a direct product of $n$ copies of the alternating group $\mathrm{Alt}_{k}$ with $k \geq 8$. Let $p$ be a prime number such that $\frac{k}{2}<p<k-2$. If $p>2 n((k-p) !)^{2 n+1}$, then $P_{G}(-1) \neq 0$.

\section{Some useful results and definitions}

Let $a$ be an integer and let $r$ be a prime number. We denote by $|a|_{r}$ the $r$-part of $a$, i.e. $|a|_{r}=r^{i}$ where $i \in \mathbb{N}$ such that $r^{i}$ divides $a$ but $r^{i+1}$ does not divide $a$. Moreover, we set $|0|_{r}=0$.

If $b=c / d$ is a rational number for some $c \in Z, d \in \mathbb{N}-\{0\}$, then we let $|b|_{r}=\frac{|c|_{r}}{|d|_{r}}$.

We record here an important result on the Möbius function of the subgroup lattice of $G$.

Lemma 5 ([5], Theorem; 4.5). Let $A$ be a finite group and $B$ a subgroup of $A$. The index $\left|N_{A}(B): B\right|$ divides $\mu_{A}(B)\left|A: B A^{\prime}\right|$.

In particular, if $X$ is an almost simple group with socle $G$ and $H$ is a subgroup of $G$, then Lemma 5 yields $|G: H|$ divides $\mu_{G}(H)\left|G: N_{G}(H)\right|$. So, in particular, $n$ divides $a_{n}(X, G)$.

We can say some more words on the Dirichlet polynomial of a monolithic primitive group $L$ with non-abelian socle $N$. Assume that $S$ is a simple component of $L$, define $X=N_{L}(S) / C_{L}(S)$ and $n=\left|L: N_{L}(S)\right|$. We have that $N \cong S^{n}$. Since $S \cong \operatorname{soc}(X)$, assume that $S \leq X$. The following result shows a connection between the Dirichlet polynomials $P_{L, N}(s)$ and $P_{X, S}(s)$. 
Theorem 6 (See [11, Theorem 5]).

$$
P_{L, N}^{(r)}(s)=P_{X, S}^{(r)}(n s-n+1)
$$

for each prime divisor $r$ of the order of $S$.

Let $X$ be $\mathrm{Sym}_{k}$ or $\mathrm{Alt}_{k}$ with $k \geq 8$. A key role in our paper is played by the Dirichlet polynomial $P_{X, S}^{(p)}(s)$, which can be expressed by an explicit formula.

Proposition 7 (See [10, Proposition 12]). Let $k \geq 8$. Let $X$ be either $\mathrm{Alt}_{k}$ or $\mathrm{Sym}_{k}$, and let $p$ be a prime number such that $\frac{k}{2}<p<k-2$. Then

$$
P_{X, S}^{(p)}(s)=\sum_{\omega \in \Psi_{k}: k_{l} \geq p} \frac{\mu(\omega)}{v(\omega) l(\omega)^{s-1}} .
$$

Here $\Psi_{k}$ is the set of partitions of $k$, i.e. non-decreasing sequences of positive integers whose sum is $k$, we have $\omega=\left(k_{1}, \ldots, k_{l}\right) \in \Psi_{k}$ for some $l \leq k, k_{1}, \ldots, k_{l} \in \mathbb{N}-\{0\}$ such that $k_{1}+\ldots+k_{l}=k$, and we define

$$
\mu(\omega)=(-1)^{l-1}(l-1) !, \quad l(\omega)=\frac{k !}{\prod_{i=1}^{l} k_{i} !}, \quad v(\omega)=\prod_{i=1}^{k} \omega_{i} !
$$

where $\omega_{i}=\left|\left\{j: k_{j}=i\right\}\right|$.

\section{The Dirichlet polynomial $P^{(p)}(s)$}

Let $X=\mathrm{Sym}_{k}$ or $\mathrm{Alt}_{k}$ for $k \geq 8$. For this section, we let $P(s)=P_{X, \operatorname{soc}(X)}(s)$. The aim of this section is to find the value $\left|P^{(p)}(1-n)\right|_{p}$ for $n \geq 2$ a natural number.

Let $p$ be a prime number such that $\frac{k}{2}<p<k-2$. By Proposition 7, we have that

$$
\begin{gathered}
P^{(p)}(1-n)=\sum_{\omega \in \Psi_{k}: k_{l} \geq p} \frac{\mu(\omega)}{v(\omega) l(\omega)^{-n}}=\sum_{\omega=\left(k_{1}, \ldots, k_{l}\right) \in \Psi_{k}: k_{l} \geq p} \frac{(-1)^{l-1}(l-1) !(k !)^{n}}{\prod_{i=1}^{k} \omega_{i} !\left(\prod_{i=1}^{l} k_{i} !\right)^{n}}= \\
=\sum_{j=0}^{k-p} g(j, n)\left(\frac{k !}{(k-j) !}\right)^{n},
\end{gathered}
$$


where

$$
g(j, n)=\sum_{\omega=\left(k_{1}, \ldots, k_{l-1}\right) \in \Psi_{j}} \frac{(-1)^{l-1}(l-1) !}{\prod_{i \geq 1} \omega_{i} !\left(\prod_{i=1}^{l-1} k_{i} !\right)^{n}}
$$

for $j \geq 0$ (the set $\Psi_{0}$ consists of the empty partition $\omega=()$, so $g(0, n)=1$ ).

Consider the polynomial

$$
f_{n}(x)=g(0, n)+\sum_{j=1}^{k-p} g(j, n)\left(\prod_{i=0}^{j-1}(x+k-p-i)\right)^{n}
$$

in $\mathrm{Q}[x]$. Clearly $f_{n}(p)=P^{(p)}(1-n)$. We want to show that $x$ divides $f_{n}(x)$ in $\mathrm{Q}[x]$, but $x^{2}$ does not divide $f_{n}(x)$ in $\mathrm{Q}[x]$. This implies that $\left|P^{(p)}(1-n)\right|_{p}=p$ if the coefficient $\alpha_{1}$ of $x$ in $f_{n}(x)$ is such that $\left|\alpha_{1}\right|_{p}=1$ (see the proof of Theorem 11).

First of all, we prove the following formula, which is very useful in order to find some properties of $g(j, n)$.

Proposition 8. If $m \geq 1$, then

$$
\sum_{j=0}^{m} \frac{g(j, n)}{((m-j) !)^{n}}=0
$$

Proof. Let us rewrite the sum in another way. Let $\omega=\left(k_{1}, \ldots, k_{l}\right) \in \Psi_{m}$. For each $h \in\{1, \ldots, l\}$ there exists $\omega^{h}=$ $\left(k_{1}, \ldots, k_{h-1}, k_{h+1}, \ldots, k_{l}\right) \in \Psi_{m-k_{h}}$. Clearly $\omega$ appears in the term $g(m, n)$ and $\omega^{h}$ appears in the term $g\left(m-k_{h}, n\right)$. In particular, the contribution of $\omega^{h}$ in $g\left(m-k_{h}, n\right)$ is

$$
\frac{(-1)^{l-1}(l-1) !}{v\left(\omega^{h}\right)\left(\prod_{i \neq h} k_{i} !\right)^{n}} .
$$

Moreover, since $v(\omega)=\left|i: k_{i}=k_{h}\right| v\left(\omega^{h}\right)$ we have that the previous expression becomes

$$
\frac{(-1)^{l-1}(l-1) !\left|i: k_{i}=k_{h}\right|}{v(\omega)\left(\prod_{i \neq h} k_{i} !\right)^{n}} .
$$

Furthermore, it is clear that if $\left(k_{1}, \ldots, k_{l^{\prime}}\right)=\tau \in \Psi_{m^{\prime}}$ for some $m^{\prime}<m$, then there exists a unique $\omega \in \Psi_{m}$ such that $\tau=\omega^{h}$ for some 
$h \in\left\{1, \ldots, l^{\prime}+1\right\}$ : indeed, there exists $h \in\left\{1, \ldots, l^{\prime}+1\right\}$ such that $k_{h-1} \leq m-m^{\prime} \leq k_{h}$ (with the convention that $k_{0}=0$ and $k_{l^{\prime}+1}=k$ ) and so $\omega=\left(k_{1}, \ldots, k_{h-1}, m-m^{\prime}, k_{h}, \ldots, k_{l^{\prime}}\right)$.

Let $H_{\omega}=\left\{h \in\{1, \ldots, l\}: h=1 \quad\right.$ or $\left.\quad k_{h-1}<k_{h}\right\} \quad$ (so $\quad\left\{k_{1}, \ldots, k_{l}\right\}=$ $\left\{k_{h}: h \in H_{\omega}\right\}$ and if $h_{1}, h_{2} \in H_{\omega}$, then $k_{h_{1}}=k_{h_{2}}$ if and only if $\left.h_{1}=h_{2}\right)$. We get

$$
\begin{aligned}
\sum_{j=0}^{m} \frac{g(j, n)}{((m-j) !)^{n}} & =\sum_{\left(k_{1}, \ldots, k_{l}\right)=\omega \in \Psi_{m}}\left(\frac{(-1)^{l} l !}{v(\omega)\left(\prod_{i=1}^{l} k_{i} !\right)^{n}}+\sum_{h \in H_{\omega}} \frac{(-1)^{l-1}(l-1) !\left|i: k_{i}=k_{h}\right|}{v(\omega)\left(\prod_{i \neq h} k_{i} !\right)^{n}} \frac{1}{\left(k_{i} !\right)^{n}}\right) \\
& =\sum_{\left(k_{1}, \ldots, k_{l}\right)=\omega \in \Psi_{m}} \frac{(-1)^{l}(l-1) !\left(l-\sum_{h \in H_{\omega}}\left|i: k_{i}=k_{h}\right|\right)}{v(\omega)\left(\prod_{i=1}^{l} k_{i} !\right)^{n}}=0
\end{aligned}
$$

since $\sum_{h \in H_{\omega}}\left|i: k_{i}=k_{h}\right|=l$ by definition.

Thanks to the recursive formula we obtained for $g(j, n)$, we can prove the following inequalities.

\section{Proposition 9. We have that}

$$
0<(-1)^{m-1} g(m-1, n) / 2<(-1)^{m} g(m, n) \leq(-1)^{m-1} g(m-1, n) \leq 1
$$

for $m \geq 1$ and $n \geq 2$.

Proof. Let us prove the proposition by induction on $m$. If $m=1$, the claim holds by definition of $g(m, n)$. Assume that $m>1$. By induction, it is enough to prove that

$$
(-1)^{m} g(m, n) / 2<(-1)^{m+1} g(m+1, n) \leq(-1)^{m} g(m, n)
$$

By Proposition 8, we have that

$$
g(m+1, n)=-\sum_{j=0}^{m} \frac{g(j, n)}{((m+1-j) !)^{n}},
$$

hence

$$
(-1)^{m+1} g(m+1, n)=(-1)^{m} g(m, n)+\sum_{j=0}^{m-1}(-1)^{m-j} \frac{(-1)^{j} g(j, n)}{((m+1-j) !)^{n}} .
$$


Let $g^{\prime}(j, n)=(-1)^{m-j} \frac{(-1)^{j} g(j, n)}{((m+1-j) !)^{n}}$. Now, by induction we have

$$
-(-1)^{j} g(j, n)<-(-1)^{j-1} g(j-1, n) / 2<0
$$

for $1 \leq j \leq m$. Hence, for $m-j$ odd, we get

$$
g^{\prime}(j, n)+g^{\prime}(j-1, n)<(-1)^{j-1} g(j-1, n) \frac{2-(m-j+2)^{n}}{2((m-j+2) !)^{n}} \leq 0
$$

for $n \geq 1$. This implies that

$$
\begin{aligned}
\sum_{j=0}^{m-1}(-1)^{m-j} \frac{(-1)^{j} g(j, n)}{((m+1-j) !)^{n}}=\alpha g^{\prime}(0, n) & \\
& +\sum_{1 \leq j \leq m-1, m-j \text { odd }} g^{\prime}(j, n)+g^{\prime}(j-1, n)<0
\end{aligned}
$$

where $\alpha=0$ if $m$ is even, $\alpha=1$ otherwise (when $m$ is odd, note that $\left.g^{\prime}(0, n)<0\right)$. So this proves that $(-1)^{m+1} g(m+1, n) \leq(-1)^{m} g(m, n)$.

Now, by $(\dagger)$, it remains to prove that

$$
\begin{aligned}
(-1)^{m+1} g(m+1, n) & -(-1)^{m} g(m, n) / 2 \\
& =(-1)^{m} g(m, n) / 2+\sum_{j=0}^{m-1}(-1)^{m-j} \frac{(-1)^{j} g(j, n)}{((m+1-j) !)^{n}}>0 .
\end{aligned}
$$

Again, by induction we have that $(-1)^{j} g(j, n) / 2<(-1)^{j+1} g(j+1, n)$ for $0 \leq j \leq m-1$. Hence, for $m-j$ even, we have that

$$
g^{\prime}(j, n)+g^{\prime}(j-1, n)>(-1)^{j} g(j, n) \frac{(m+2-j)^{n}-2}{((m+2-j) !)^{n}} \geq 0
$$

for $n \geq 1$. Moreover, we get

$$
(-1)^{m} g(m, n) / 2+g^{\prime}(m-1, n)>(-1)^{m} g(m, n) \frac{2^{n-2}-1}{2^{n-1}} \geq 0
$$

for $n \geq 2$. This implies that

$$
\begin{aligned}
(-1)^{m} g(m, n) / 2+\sum_{j=0}^{m-1}(-1)^{m-j} & \frac{(-1)^{j} g(j, n)}{((m+1-j) !)^{n}} \\
= & \beta g^{\prime}(0, n)+(-1)^{m} g(m, n) / 2+g^{\prime}(m-1, n) \\
& +\sum_{1 \leq j \leq m-2, m-j \text { even }} g^{\prime}(j, n)+g^{\prime}(j-1, n)>0
\end{aligned}
$$


where $\beta=1$ if $m$ is even, $\beta=0$ otherwise (when $m$ is even, note that $\left.g^{\prime}(0, n)>0\right)$. So this proves that $(-1)^{m+1} g(m+1, n)>(-1)^{m} g(m, n) / 2$.

Now, we are ready to prove the main result of this section.

Proposition 10. Let $n \geq 2$. We have that $x$ divides $f_{n}(x)$ in $\mathrm{Q}[x]$, but $x^{2}$ does not divide $f_{n}(x)$ in $\mathrm{Q}[x]$.

Let $m=k-p$. To prove that $x$ divides $f_{n}(x)$ is enough to show that

$$
f_{n}(0)=\sum_{j=0}^{m} g(j, n)\left(\frac{m !}{(m-j) !}\right)^{n}=0,
$$

which follows from Proposition 8. In order to prove that $x^{2}$ does not divide $f_{n}(x)$ we may show that the coefficient of $x$ in $f_{n}(x)$ does not vanish. It is easy to realize that the coefficient of $x$ in $f_{n}(x)$ is:

$$
\sum_{j=0}^{m} n g(j, n)\left(\frac{m !}{(m-j) !}\right)^{n} \sum_{i=0}^{j-1} \frac{1}{m-i}
$$

By Proposition 8, we can subtract

$$
\sum_{j=0}^{m} n g(j, n)\left(\frac{m !}{(m-j) !}\right)^{n} \sum_{i=0}^{m-1} \frac{1}{m-i}=0
$$

from $(\dagger)$, hence proving that $(\dagger)$ does not vanish is equivalent to prove that

$$
\sum_{j=0}^{m-1} g(j, n)\left(\frac{m !}{(m-j) !}\right)^{n} \sum_{i=j}^{m-1} \frac{1}{m-i} \neq 0 .
$$

Let

$$
h_{a, b}(m, n)=\sum_{j=a}^{b} g(j, n)\left(\frac{m !}{(m-j) !}\right)^{n} \sum_{i=j}^{m-1} \frac{1}{m-i} .
$$

Let $0 \leq j \leq m-2$. Note that

$$
\left(\frac{m !}{(m-j-1) !}\right)^{n} \sum_{i=j+1}^{m-1} \frac{1}{m-i} \geq 2\left(\frac{m !}{(m-j) !}\right)^{n} \sum_{i=j}^{m-1} \frac{1}{m-i}>0
$$

since

$$
(m-j)^{n} \sum_{i=j+1}^{m-1} \frac{1}{m-i} \geq 2 \sum_{i=j}^{m-1} \frac{1}{m-i}>0 .
$$


Hence, by Proposition 9 we get

$$
\begin{aligned}
(-1)^{j+1} g(j+1, n)\left(\frac{m !}{(m-j-1) !}\right)^{n} \sum_{i=j+1}^{m-1} & \frac{1}{m-i}> \\
& (-1)^{j} g(j, n)\left(\frac{m !}{(m-j) !}\right)^{n} \sum_{i=j}^{m-1} \frac{1}{m-i},
\end{aligned}
$$

i.e. $(-1)^{j+1} h_{j, j+1}(m, n)>0$. This implies that $h_{0, m-1}(m, n)<0$ if $m$ is even, and $h_{3, m-1}(m, n)>0$ if $m$ is odd. Since $g(0, n)=-g(1, n)=1$ and $g(2, n)=1-2^{-n}$, it is easy to see that $h_{0,2}(m, n)>0$ (for $n \geq 2$ and $m \geq 3$ ). Thus we conclude that

$$
h_{0, m-1}(m, n)=h_{0,2}(m, n)+h_{3, m-1}(m, n)>0
$$

if $m$ is odd. The proof is complete.

We can finally prove the main theorem.

THEOREm 11. Let $k \geq 8$ and let $p$ be a prime number such that $k / 2<p<k-2$. Assume that $p>n((k-p) !)^{n+1}$. Then

$$
\left|P^{(p)}(-n+1)\right|_{p}=p .
$$

Proof. Let $m=k-p$. Let $f_{n}(x)=\alpha_{0}+\alpha_{1} x+\ldots+\alpha_{t} x^{t}$ for some $\alpha_{i}=a_{i} / b_{i} \in \mathrm{Q}$ with $\left(a_{i}, b_{i}\right)=1$ (if $a_{i}=0$, let $b_{i}=1$ ) and $t \in \mathbb{N}$.

By the proof of Proposition 10 we have that $\alpha_{1}=-n h_{0, m-1}(m, n)$. Moreover, we have seen that $(-1)^{m-1} h_{0, m-1}(m, n)>0$ and $(-1)^{m-1} h_{0, m-2}(m, n)<0$, hence $\left|h_{m-1, m-1}(m, n)\right|>\left|h_{0, m-1}(m, n)\right|$. So we have

$$
\left|\alpha_{1}\right| \leq n\left|h_{m-1, m-1}(m, n)\right|=n|g(m-1, n)|(m !)^{n} \leq n(m !)^{n}
$$

being $|g(m-1, n)| \leq 1$ (by Proposition 9).

Now, we want to show that $\left|P^{(p)}(-n+1)\right|_{p}=p$. Note that since $p>k-p$ and $b_{i}$ divides $(k-p) !=m$ ! (by definition of $f_{n}(x)$ ), we have that $\left|\alpha_{i}\right|_{p}=\left|a_{i}\right|_{p}$. By Proposition 10 we have that $\alpha_{0}=0$, hence $\left|P^{(p)}(-n+1)\right|_{p}=$ $\left|f_{n}(p)\right|_{p} \geq p$. For a contradiction, assume that $\left|P^{(p)}(-n+1)\right|_{p}>p$. Then $\left|f_{n}(p)\right|_{p} \geq p^{2}$, hence $\left|\alpha_{1}\right|_{p} \geq p$, so

$$
\left|a_{1}\right| \geq\left|\alpha_{1}\right|_{p} \geq p>n(m !)^{n+1} \geq\left|\alpha_{1}\right| m ! \geq\left|\alpha_{1}\right|\left|b_{1}\right|=\left|a_{1}\right|,
$$

a contradiction. 
When the number $k-p$ is small, we know explicitly the coefficient of $x$ in $f_{n}(x)$. For example, we have that $\left|P^{(p)}(-n+1)\right|_{p}=p$ if $p$ does not appear in Table 1 (for $k-p \leq 7$ and $n \in\{2,4,6\}$ ). Further calculations can be done, but the coefficient of $x$ in $f_{n}(x)$ grows very quickly and it has very large prime factors (for example, if $k-p=35$, the coefficient of $x$ in $f_{2}(x)$ has a prime divisor of 50 digits).

TABLE 1. Exceptions for $p$.

\begin{tabular}{cccc}
\hline$k-p$ & $n=2$ & $n=4$ & $n=6$ \\
\hline 3 & 23 & 31,53 & 109,617 \\
4 & 677 & 1047469 & $19,31,5051$ \\
5 & 71,103 & 643,148579 & 3889,595523689 \\
6 & 7,13 & $23,269,89714671$ & $2357,4584299,35430211$ \\
7 & 863897 & 181,2005732476817 & 70353197,1633443829219 \\
\hline
\end{tabular}

However, there are many prime numbers between $k / 2$ and $k-2$ if $k$ is big enough. For example, we have the following.

Proposition 12. Let $8 \leq k \leq 10^{6}$. If $k \neq 13$, then there exists a prime number $p$ such that $\left|P^{(p)}(-1)\right|_{p}=p$.

\section{The main result}

We can now prove the main result.

THEOREM 13. Let $G$ be a monolithic primitive group with socle isomorphic to $\mathrm{Alt}_{k}^{n}$ for $k \geq 8$. Let $p$ be a prime number such that $k / 2<p<k-2$.

(1) If $p>2 n((k-p) !)^{2 n+1}$, then $P_{G, \operatorname{soc}(G)}(-1) \neq 0$.

(2) If $n=1$ and $k-p \leq 35$, then $P_{G, \operatorname{soc}(G)}(-1) \neq 0$.

(3) If $n=1$ and $8 \leq k \leq 10^{7}$, then $P_{G, \operatorname{soc}(G)}(-1) \neq 0$.

Proof. By Theorem 6, we have that

$$
\left|P_{G, \operatorname{soc}(G)}^{(p)}(-1)\right|_{p}=\left|P_{X, \operatorname{soc}(X)}^{(p)}(1-2 n)\right|_{p},
$$

where $X=N_{G}(S) / C_{G}(S)$ and $S$ is a simple component of $G$. By Theorem 11 , if $p>2 n((k-p) !)^{2 n+1}$, then $\left|P_{X, \operatorname{soc}(X)}^{(p)}(1-2 n)\right|_{p}=p$. By Lemma 5 , we have 
that $l$ divides $a_{l}(G, \operatorname{soc}(G))$ for $l \geq 1$, hence

$$
\left|P_{G, \operatorname{soc}(G)}(-1)-P_{G, \operatorname{soc}(G)}^{(p)}(-1)\right|_{p} \geq p^{2},
$$

thus we get the claim.

If $n=1$ and $k-p \leq 35$, the coefficient of $x$ in $f_{n}(x)$ is known, so by direct computation, there exists a prime number $p^{\prime}$ (not necessarily different from $p$ ) such that $k / 2<p^{\prime}<k-2$ such that $\left|P_{X, \operatorname{soc}(X)}^{\left(p^{\prime}\right)}(-1)\right|_{p^{\prime}}=p^{\prime}$ (except when $k=13$ ). Arguing as above, we get the claim (using a direct computation for $k=13$ ). Finally, if $n=1$ and $8 \leq k \leq 10^{6}$, arguing as above, by Proposition 12 we have the claim.

\section{REFERENCES}

[1] N. Boston, A probabilistic generalization of the Riemann zeta function, Analytic Number Theory, 1 (1996), pp. 155-162.

[2] K. S. BRown, The coset poset and the probabilistic zeta function of a finite group, J. Algebra, 225 (2000), pp. 989-1012.

[3] E. Detomi - A. Lucchini, Crowns and factorization of the probabilistic zeta function of a finite group, J. Algebra, 265 (2003), pp. 651-668.

[4] W. Gaschütz, Zu einem von B. H. und H. Neumann gestellten Problem, Math. Nachr., 14 (1955), pp. 249-252.

[5] T. Hawkes - M. IsaAcs - M. ÖZAYdin, On the Möbius function of a finite group, Rocky Mountain Journal, 19 (1989), pp. 1003-1034.

[6] A. Mann, Positively finitely generated groups, Forum Math., 8 (1996), pp. 429-459.

[7] M. Patassini, The Probabilistic Zeta function of $\operatorname{PSL}_{2}(q)$, of the Suzuki groups ${ }^{2} \mathrm{~B}_{2}(q)$ and of the Ree groups ${ }^{2} \mathrm{G}_{2}(q)$, Pacific J. Math., 240 (2009), pp. $185-200$.

[8] M. Patassini, On the Dirichlet polynomial of the simple group of Lie type, Università di Padova, 2011, http://paduaresearch.cab.unipd.it/3272/1/Phd_Thesis.pdf,

[9] M. Patassini, On the (non-)contractibility of the order complex of the coset poset of a classical group, J. Algebra, 343 (2011), pp. 37-77.

[10] M. Patassini, Recognizing the non-Frattini abelian chief factors of a finite group from its Probabilistic Zeta function, Accepted by Comm. Algeb., 2011.

[11] P. Jiménez Seral, Coefficient of the Probabilistic Zeta function of a monolithic group, Glasgow J. Math., 50 (2008), pp. 75-81.

Manoscritto pervenuto in redazione il 9 Settembre 2011. 